Supporting Information for

\title{
Supramolecular Chirality in Achiral Polyfluorene: Chiral Gelation, Memory of Chirality and Chiral Sensing Property
}

Yin Zhao, ${ }^{\dagger}$ Nor Azura Abdul Rahim, ${ }^{\dagger}$ Yijun Xia ${ }^{\dagger}$ Michiya Fujiki, ${ }^{\dagger}$ Bo Song, ${ }^{\dagger}$

Zhengbiao Zhang, ${ }^{\dagger}$ Wei Zhang, ${ }^{*}{ }^{\dagger}$ and Xiulin $\mathrm{Zhu}^{\dagger}$

${ }^{\dagger}$ State and Local Joint Engineering Laboratory for Novel Functional Polymeric Materials, Jiangsu Key Laboratory of Advanced Functional Polymer Design and Application, College of Chemistry, Chemical Engineering and Materials Science, Soochow University, Suzhou 215123, China.

ॠGaduate School of Materials Science, Nara Institute of Science and Technology, 8916-5 Takayama, Ikoma, Nara 630-0192, Japan 
(a)

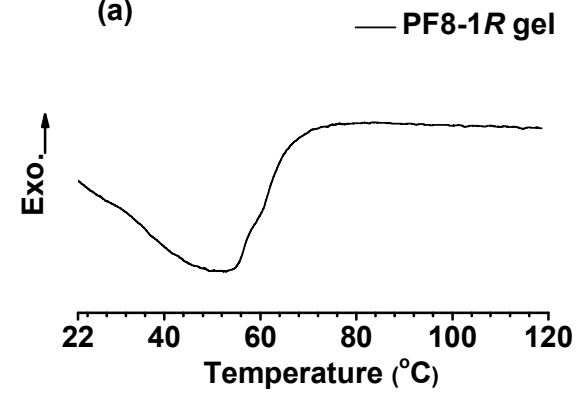

(c)

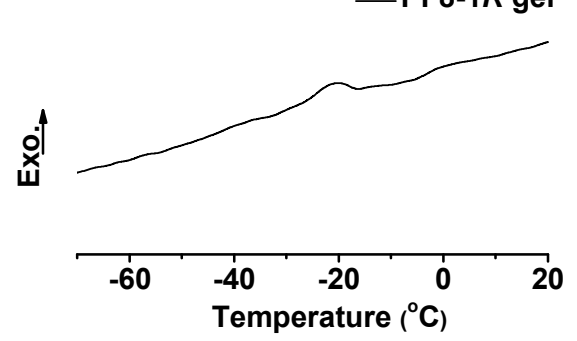

(b)

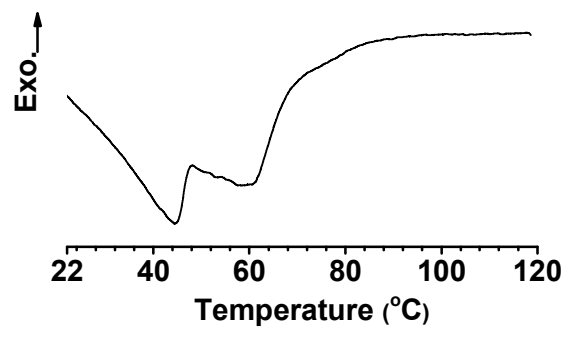

(d)

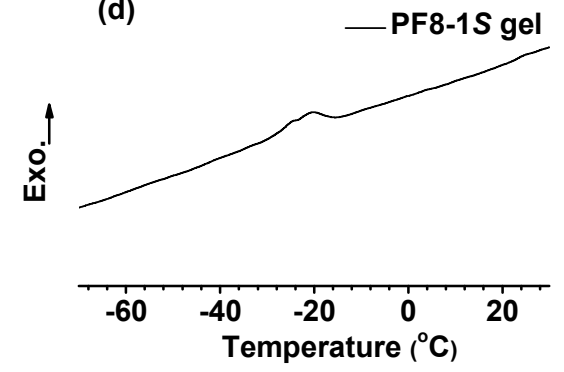

Figure S1. DSC thermograms of PF8 gels in neat $1 R$ and $1 S$ obtained in heating ((a) and (b)) and subsequent cooling ((c) and (d)) cycles.
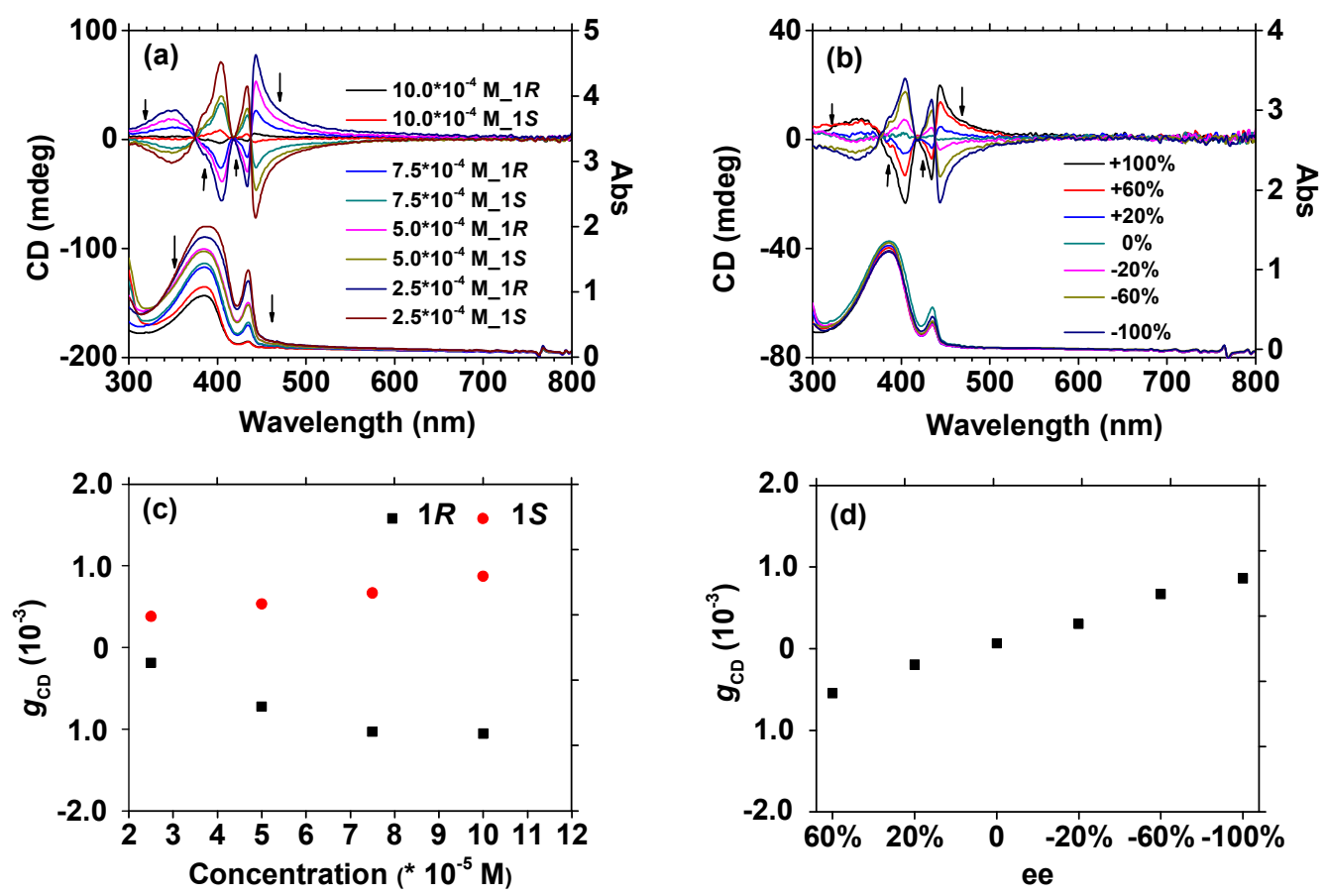

Figure S2. UV and CD spectra as a function of the concentration and enantiopurity of 
limonene. (a) In pure $1 R$ or $1 S$, the concentration varies from 2.5 to $10 \times 10^{-4} \mathrm{M}$. (b) With the same polymer concentration $\left(5 \times 10^{-4} \mathrm{M}\right)$, the enantiopurity of limonene changes from $+100 \%(1 R)$ to $-100 \%(1 S)$. The $g_{\mathrm{CD}}$ value (at $443 \mathrm{~nm}$ ) of PF8 as a function of aging time at (c) different concentrations and (d) different enantiopurity of limonene.
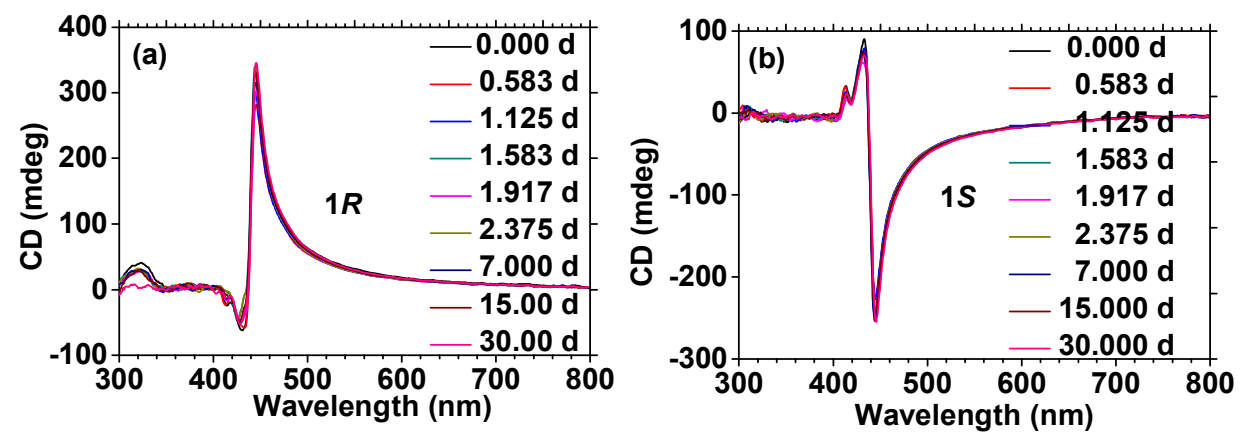

Figure S3. CD spectra monitored the stability of PF8 aggregates formed at $-20{ }^{\circ} \mathrm{C}$ in pure (a) $1 R$ or (a) $1 S$.
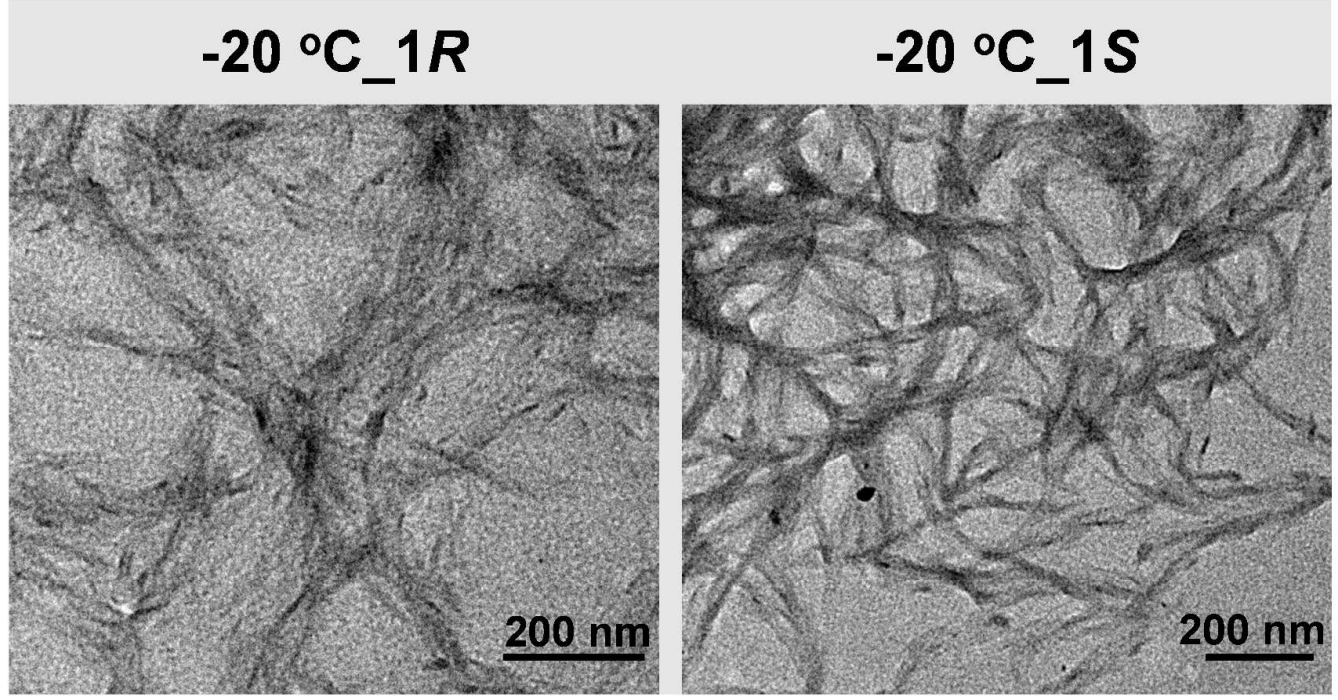

Figure S4. TEM images of PF8 assemblies formed at $-20{ }^{\circ} \mathrm{C}$ for $40 \mathrm{~h}$ in $1 R$ (left) and (b) $1 S$ (right). 

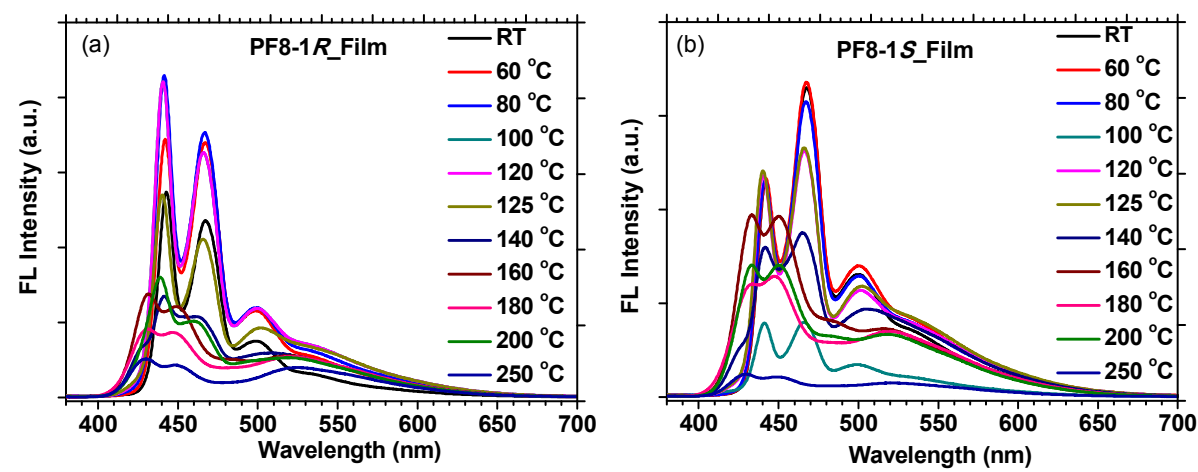

Figure S5. Temperature dependence of the fluorescence emission spectra of (a) PF8_1R and (b) PF8_1S films. The films were spin-coated from $1 R$ and $1 S$ solutions $(2.0 \mathrm{mg} / \mathrm{mL})$ which were cooled at $-20{ }^{\circ} \mathrm{C}$ for $40 \mathrm{~h}$ previously, and dried at $60{ }^{\circ} \mathrm{C}$ under vacuum overnight. Each temperature was retained for $10 \mathrm{~min}$.

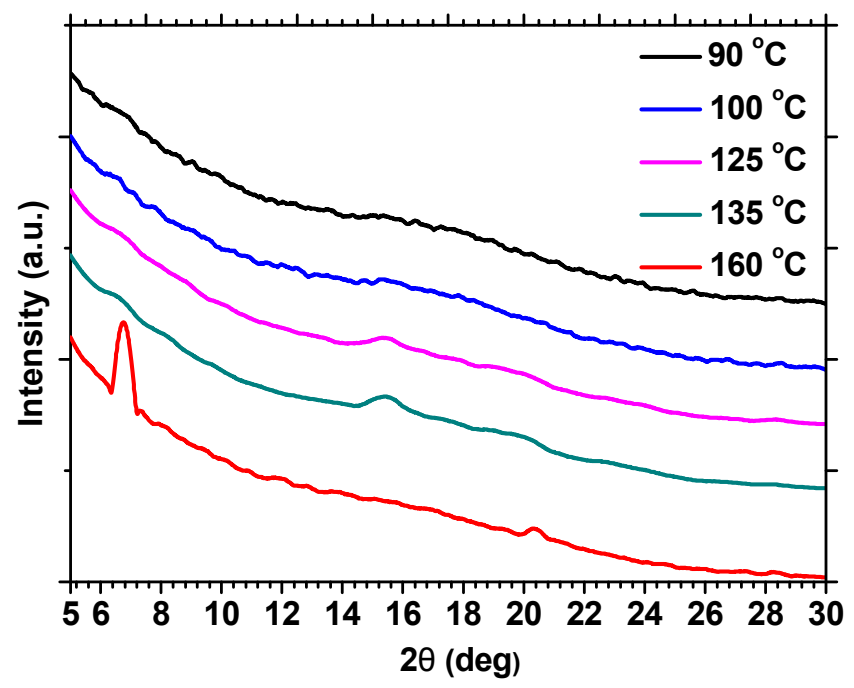

Figure S6. XRD profile of the PF8 film drop-cast from PF8 aggregates obtained at $-20{ }^{\circ} \mathrm{C}$. Below $80{ }^{\circ} \mathrm{C}$, the film was amorphous. When elevating the temperature subsequently, the cast film displays crystalline $\alpha$-phase scattering peaks. 


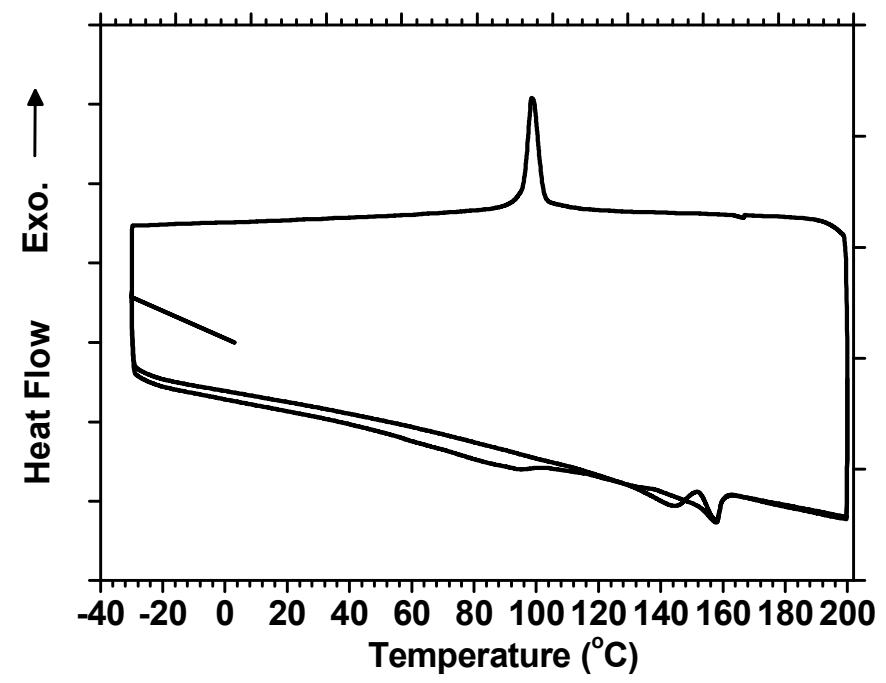

Figure S7. DSC heating traces of PF8-1 $R$ xerogel. DSC heating traces showed the glass transition temperature around $100{ }^{\circ} \mathrm{C}$, and subsequent transformation into $\alpha$-phase that melts near $160{ }^{\circ} \mathrm{C}$. DSC cooling traces showed crystallization at rather low temperature near $100{ }^{\circ} \mathrm{C}$. Samples prepared from PF8 xerogel with a typical mass of about $5 \mathrm{mg}$ were encapsulated in sealed aluminum pans.

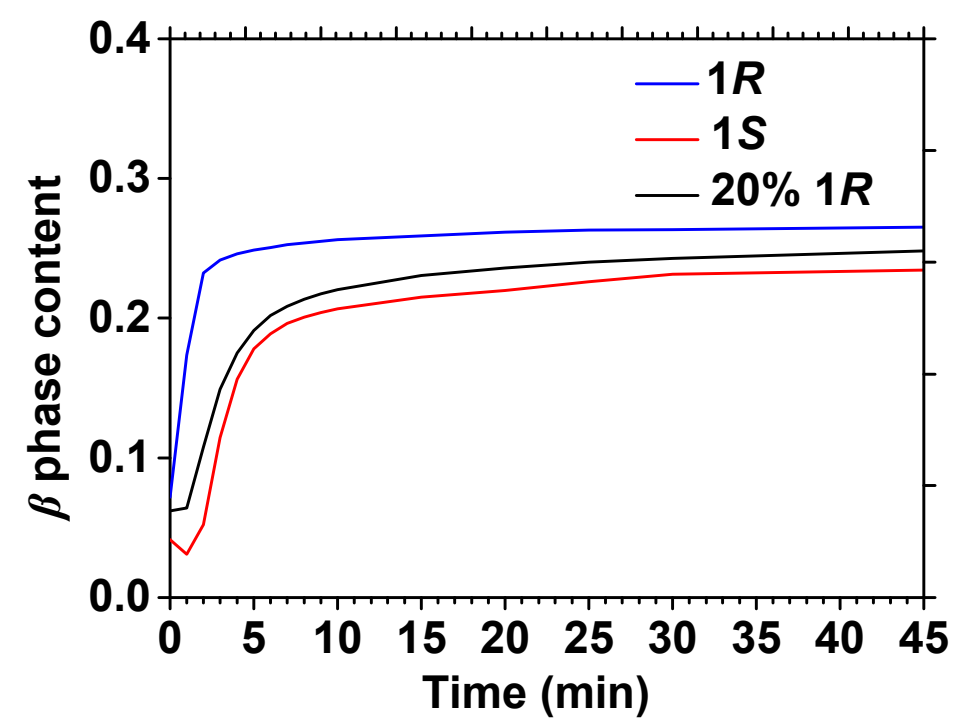

Figure S8. $\beta$-phase contents of PF8 molecules formed in different enatiopurity of limonene was compared. 